\title{
EDITORIAL
}

\section{Vascular compromise secondary to fillers injection: Minimizing sequelae}

\author{
Serag Monier Zidan
}

Faculty of Medicine, Al-Azhar University, Egypt

$\mathrm{T}$ The use of filler injections for facial rejuvenation and sculpturing has become a keystone of esthetic medicine. According to American Society for Aesthetic Plastic Surgery (ASAPS), filler injections are the second most common minimally invasive procedure performed by plastic surgeons in $2012^{[1]}$.

Although injection of fillers is a minimally invasive procedure, it does not come without complications. Echymosis, infections, edema, and unsatisfactory esthetic outcomes are common complications of filler injections $^{[2]}$.

Skin necrosis, which is secondary to vascular compromise, is a rare but stressful complication of filler injections, and is more likely to result in permanent sequelae. It is caused by the interruption of cutaneous blood supply, leading to the decrease or loss of cutaneous perfusion.

Occlusion of cutaneous blood vessels can be due to extravascular compression by filler or associated inflammation and edema. It can be also caused by the embolization of filler material or damage to the wall of blood vessels ${ }^{[3]}$.

In most cases, vascular compression by filler material occurs immediately after injection. However, it may present later if compression develops as a result of increasing edema and tissue pressure ${ }^{[4]}$.

Pathophysiology of cutaneous vascular occlusion begins with initial blanching. This is followed by mottled discoloration called livedo reticularis. The resulting ischemia produces a dusky discoloration and finally irreversible skin necrosis occurs ${ }^{[5]}$.

The symptoms of vascular compromise usually start with immediate and severe pain. Clinically there is blanching, duskiness, and ecchymosis. In some cases when there is delayed compression of vessels by the product, symptoms are minimal ${ }^{[1]}$.

The presence of immediate skin blanching and severe pain suggests arterial injury. On the other hand, venous occlusion is associated with persistent dull, aching pain and swelling, and with violaceous discoloration of the affected area ${ }^{[6]}$.

Several precautions should be taken to minimize the risk of vascular compromise associated with filler injections. These precautions are of utmost importance when injecting into high-risk anatomical areas such as the glabella or nasolabial folds.

Precautions start with the taking of patients' detailed history to assess previous treatment with fillers or cosmetic surgeries in the area to be treated. For example, rhinoplasty surgery alters blood supply to nasal skin, making subsequent filler injections to nose or nasolabial folds more risky.

The use of small-gauge (30- or 32-G) needles (aspirating before injection if possible), with material injected during needle withdrawal, and with low volumes of product in two or more treatment sessions could reduce the risk of vascular occlusion. The usage of injection cannulas with blunt tip and larger gauge (25- or even $22-\mathrm{G}$ cannulas) is another strategy preferred by some experts. An awareness of injection plane may also reduce this complication ${ }^{[6]}$.

Appropriate treatment should be initiated immediately upon suspicion of vascular compromise. Injection should be stopped, and the area of injection should be massaged immediately. In addition, warm compresses should be applied to increase vasodilatation.

Hyaluronidase is recommended to break down and hydrolyze hyaluronic acid. It has been proposed to be beneficial even in cases caused by non-hyaluronic acid fillers, as it possesses edema-reducing benefits and theoretically reduces occluded vessel pressure. The dose of hyaluronidase ranges from 10 to 30 units $^{[7]}$.

Use of topical $2 \%$ nitroglycerin paste is beneficial in cases presenting early. It can be applied initially every

Copyright () 2018 Zidan SM. This is an Open Access article distributed under the terms of the Creative Commons Attribution-NonCommercial 4.0 International License (http://creativecommons.org/licenses/by-nc/4.0/), permitting all non-commercial use, distribution, and reproduction in any medium, provided the original work is properly cited. 
one to two hours under observation. Nitroglycerin paste treatment can be continued at home three times daily, as proposed by some authors, but patient should be advised to stop using it in case of dizziness ${ }^{[3]}$.

Other treatments include systemic or topical steroids to reduce the associated inflammation and edema. Other options that have been proposed but with unproven efficacy include aspirin, intravenous prostaglandins, filler removal via puncture, low-molecular-weight heparin, and sildenafil. The use of hyperbaric oxygen is controversial $^{[3,6,7]}$.

If necrosis does occur, further treatment is directed to enhance healing in order to minimize scarring. Gentle debridement is beneficial to reduce healing time. A variety of topical wound dressings can be used to achieve wound hydration, antimicrobial action and debriding activity. Systemic antibiotics are used only if there is evidence of infection. Reconstructive procedures may be needed in cases of skin loss not amenable to healing by secondary intention.

Skin necrosis secondary to fillers injection, as any complication in medical practice, is not completely preventable. However, its incidence can be lowered by probable precautions and its sequelae can be minimized by early active management. Legal issues should also be considered by proper patient counseling and informed consent.

\section{Conflict of interest}

The author declares no potential conflict of interest with respect to the research, authorship, and/or publication of this article.

\section{References}

1. Ozturk CN, Li Y, Tung R, Parker L, Piliang MP, et al. Complications following injection of soft-tissue fillers. Aesthet Surg J 2013; 33(6): 862-877. doi: 10.1177/1090$820 X 13493638$.

2. Girolamo MD, Mattei M, Signore A, Grippaudo FR. MRI in evaluation of facial dermal fillers in normal and complicated cases. Euro Radiol 2015; 25(5): 1431-1442. doi: 10.1007/s00330-014-3513-2.

3. Beleznay K, Humphrey S, Carruther JDA, Carruthers A. Vascular compromise from soft tissue augmentation. J Clin Aesthet Dermatol 2014; 7(9): 37-43.

4. Hirsch RJ, Lupo M, Cohen JL, Duffy D. Delayed presentation of impending necrosis following soft tissue augmentation with hyaluronic acid and successful management with hyaluronidase. J Drugs Dermatol 2007; 6(3): 325-328.

5. Kim JH, Ahn DK, Jeong HS, Suh IS. Treatment algorithm of complications after filler injection: Based on wound healing process. J Korean Med Sci 2014; 29(Suppl 3): S176-S182. doi: 10.3346/jkms.2014.29.S3.S176.

6. Sclafani AP, Fagien S. Treatment of injectable soft tissue filler complications. Dermatol Surg 2009; 35(Suppl 2): 1672-1680. doi: 10.1111/j.1524-4725.2009.01346.x.

7. Dayan SH, Arkins JP, Mathison CC. Management of impending necrosis associated with soft tissue filler injections. J Drugs Dermatol 2011; 10(9): 1007-1012.

Keywords: fillers; filler complications; hyaluronidase

Citation: Zidan SM, Vascular compromise secondary to fillers injection: Minimizing sequelae. J Surg Dermatol 2018; 3(2): 112; http://dx.doi.org/10.18282/jsd.v2.i1.112.

Received: $23^{\text {rd }}$ June 2018; Published Online: $8^{\text {th }}$ July 2018

Correspondence to: Serag Monier Zidan, Faculty of Medicine, Al-Azhar University, Egypt; prs.zidan@gmail.com 